\title{
Efficient Transmission of 2D Chaotic Maps Encrypted Images with DWT-Based SC-FDMA LTE System
}

\author{
Elhadi Mehallel ${ }^{1,2^{*}}$, Djamel Abed ${ }^{2}$, Ammar Bouchemel $^{2}$ \\ ${ }^{1}$ Faculty of Science and Technology, University of Ziane Achour, 17000 Djelfa, P.O.B. 3117, Algeria \\ 2 LABCAV advanced control laboratory, Faculty of Science and Technology, University 8th May 1945, 24000 Guelma, P.O.B. 401, Algeria \\ * Corresponding author, e-mail: e.mehallel@univ-djelfa.dz
}

Received: 11 March 2021, Accepted: 03 August 2021, Published online: 13 December 2021

\begin{abstract}
The single-carrier frequency division multiple access (SC-FDMA) is a promising technique that has been adopted as an uplink transmission scheme in the long-term evolution (LTE) cellular system. This is attributed to its advantages such as the low peak-to-average power ratio (PAPR) and the utilization of frequency-domain equalizers to resolve the problem of inter-symbol interference (ISI). In this paper, a Discrete Wavelet Transform (DWT) based SC-FDMA system is proposed for the effective transmission of encrypted images. The 2D Chaotic baker map encryption algorithm has been used to encrypt images to enhance their security during transmission via SC-FDMAbased systems. The performance of the process of encrypted image transmission using the 2D Chaotic baker map algorithm with wavelet transform-based SC-FDMA (DWT SC-FDMA) system is evaluated in terms of different performance metrics, with comparison to Discrete Fourier Transform SC-FDMA (DFT SC-FDMA) and, Discrete Cosine Transform SC-FDMA (DCT SC-FDMA) systems. The viability of the proposed scheme was tested with different wireless channel models and different subcarriers mapping schemes. Experimental results show that the proposed method of the encrypted image transmission via the DWT SC-FDMA system provides a remarkable performance gain compared to the other versions of the SC-FDMA system in terms of the PSNR, and the BER metrics in the wireless channel models. It also demonstrates the effectiveness of the proposed scheme and adds a degree of encryption to the transmitted images through the wireless channels.
\end{abstract}

Keywords

DWT, PSNR, SC-FDMA system, 2D Chaotic baker map, encrypted image transmission

\section{Introduction}

The Single-Carrier Frequency-Division Multiple Access (SC-FDMA) technique has been a promising solution for high data-rate uplink transmission. It has been proposed as an alternative to Orthogonal Frequency-Division Multiple Access (OFDMA) in the long-term evolution (LTE) standard, which is used for downlink transmission [1, 2]. In other hand, the major advantages of the SC-FDMA system are low peak-to-average power ratio (PAPR) due to lower signal envelope variations in the SC-FDMA transmitted signal, and the power efficiency is higher than that of the OFDMA system [3, 4]. Because of this reasons, the LTE cellular system adopts the SC-FDMA on the uplink communication to exploit this low PAPR advantage, which permits the use of an efficient power amplifier in order to preserve the battery life of the mobile terminal [5]. There are two modes of resource allocation for the SC-FDMA system to choose subcarriers for each user. These are the
Interleaved-FDMA (IFDMA) mode, which assigns equidistant subcarriers to each user, and the Localized-FDMA (LFDMA), which assigns groups of contiguous subcarriers to a particular user [6]. Usually, LTE adopts the traditional DFT SC-FDMA system for uplink transmission to reduce the PAPR of the transmitted signals [7]. The traditional SC-FDMA system has the same overall complexity as the OFDMA system with a similar throughput performance. Several approaches have been proposed as alternatives to the traditional DFT-SC-FDMA systems like Discrete Cosine Transform OFDM (DCT-SC-FDMA) and Discrete Wavelet Transform DWT (DWT-SC-FDMA) [8, 9]. DWT has become a more popular tool over traditional DFT and DCT in most signal processing and communication applications mainly because of its advantages of multi-resolution signal analysis with better localization in both time and frequency domain and provides high spectral efficiency and 
suitable pulse shaping properties. DWT can be regarded as a multi-resolution decomposition of a signal into its components in different frequency bands and analyze each component with a resolution matched to its scale [10].

Due to the rapid development of internet technology and multimedia services, wireless transmission of images and video can be easily performed over multipath channels. Transmission of images and multimedia with a recent variant of Orthogonal Frequency Division Multiplexing (OFDM) technology has attracted the attention of several researchers $[11,12]$. In the literature, different and efficient image transmission schemes over OFDM and multicarrier code division multiple access (MC-CDMA) systems have attracted the attention of several researchers [13, 14]. Recently, the issue of high-efficiency image transmission over OFDMA and SC-FDMA systems was studied in $[15,16]$. In [16], the issue of wireless image transmission over three different versions of the OFDMA system is investigated for different transmission scenarios. Additionally, the performance of image transmission over two different structures namely: DFT SC-FDMA and DCT SC-FDMA was implemented and studied in order to select the proper technique for efficient image transmission [17].

With the rapid development of wireless communication and multimedia applications, in particular images, information security has become an important prime issue and data encryption is one of the most important means of ensuring security. On the other hand, image transmission over wireless communication systems has prompted new problems with security and confidentiality. Hence, an efficient cryptosystem is therefore needed to ensure the security of the image transmission system with higher reliability of communication. In general, two main approaches are used for the protection of digital images. The first one is information hiding such as digital watermarking of images. The second is image encryption, which includes conventional encryption schemes such as chaotic encryption [18-20]. The traditional image encryption method considers the plaintext as the original data stream, due to some intrinsic features of images, such as bulk data size and high correlation between pixels. Traditional encryption algorithms, such as Advanced Encryption Standard (AES) or Data Encryption Standard (DES) are generally not suitable for practical image encryption [21]. Recently, with the development of nonlinear dynamics and chaos theory, some common features, such as sensitivity to variables and parameters, between chaos and cryptography have been revealed and exploited [22]. It has drawn increasing efforts to use chaotic systems to improve the security of communications [23]. The use of chaotic maps for image encryption will offer numerous advantages as compared with traditional methods, such as easy implementation, lower mathematical complexity, faster encryption speed, and a higher degree of security [24]. Many image encryption schemes based on chaotic maps have been developed in recent years [25]. However, most of these schemes are using generally $1 \mathrm{D}$ chaotic maps, which has security weaknesses [26]. Several encryption schemes based on two-dimensional (2D) Chaotic maps have been proposed to improve the security of image data in recent years [27]. These encryption schemes use permutation plus diffusion in image encryption to compose very good encryption schemes with a higher degree of image security, lower time complexity, and faster encryption speed. Therefore, a suitable and secure encryption scheme is required to employ it in real-time applications.

The main object of this paper is to propose an efficient scheme for encrypted image transmission over the SC-FDMA system based on Wavelet Discrete Transforms (DWT). The performances of encrypted image transmission by the 2D Chaotic baker map over the DWT-SCFDMA system are investigated and compared to both DFTSC-FDMA and DCT-SC-FDMA systems. In addition, the Bit-Error-Rate (BER) and the Peak-Signal-to-Noise Ratio (PSNR) performances of the received image over DFTSC-FDMA, DCT-SC-FDMA, and proposed DWT-SCFDMA systems are evaluated and compared for different wireless channels and subcarriers mapping schemes. The obtained results show that the DWT-based SC-FDMA system achieves the best PSNR and BER performances than the conventional SC-FDMA systems.

The rest of this paper is organized as follows. In Section 2, the image transmission over the different SC-FDMA systems will be discussed. Section 3 presents the 2D Chaotic baker maps algorithm for image encryption. In Section 4, the DWT-based SC-FDMA architecture for encrypted image transmission will be explained. Section 5 illustrates the simulation results. Section 6 gives the conclusions.

\section{SC-FDMA systems model for image transmission}

This section describes the different schemes developed for image transmission over SC-FDMA systems include DFTSC-FDMA system and DCT-SC-FDMA system. 


\subsection{The conventional Discrete Fourier Transform-based SC-FDMA system}

At the transmitter, the grayscale image is transformed primarily into a 2D binary form suitable to be transmitted easily by the SC-FDMA-based system (Fig. 1). After that, the binary bits are encoded and then modulated using a modulation scheme. Let $x_{n}$ denote the source symbols in time-domain, and $X_{k}$ denote the obtained frequency symbols after DFT block. Then, the obtained signal after the output of DFT modulator is given by

$X_{k}=\sum_{n=0}^{N-1} x_{n} e^{-\frac{j 2 \pi}{N} n k}, n=0, \ldots, N-1$.

The block of the $N$ symbols are then mapped to $M(M>N)$ orthogonal sub-carriers in the frequency domain using the interleaved or localized subcarriers mapping modes, where $M=Q \times N$ is the number of sub-carriers allocated to each user, and $Q$ denotes the maximum number of users. After the mapping process, the IDFT is applied to transform the frequency symbols into the time-domain before the transmission. The inverse transform generates a time-domain symbol $y_{m}$. Therefore, the output signal after IDFT can be written as follows:

$y_{m}=\frac{1}{M} \sum_{k=0}^{M-1} Y_{k} e^{\frac{j 2 \pi}{M} k m}, k=0, \ldots, M-1$,

where $M$ is the FFT length and $Y_{k}$ signifies the frequency domain samples after the subcarriers mapping process. To limit the Inter-Block Interference (IBI) caused by multipath propagation, a Cyclic-Prefix (CP) is inserted at the start of each block on the transmitter side. Finally, the signal is passed through a digital-to-analog conversion (DAC) and then transmitted over a wireless channel.

At the receiver side, the $\mathrm{CP}$ is removed and then the received signal is transformed into the frequency domain by using DFT. After the de-mapping process, the frequency samples are then passed through the FrequencyDomain-Equalization-(FDE) to the limit of the ISI. Then the N-point IDFT in the receiver transforms equalized symbols back into the time domain. The demodulation process recuperates the original data, which is then passed through the decoder. Finally, the data reconstruction stage at the receiver, which is used for converting the binary format to restore the original image.

\subsection{The Discrete Cosine Transform-based SC-FDMA system}

The main advantage of the DCT over the DFT is its excellent spectral energy concentration property, which limits the effects of inter-symbol interference (ISI). In addition, the DCT can be used instead of the DFT to facilitate operations based on real arithmetic [2].

The block diagram of the DCT-SC-FDMA system for image transmission is shown in Fig. 2. Therefore, the architecture of the DCT-based SC-FDMA is similar to that of the DFT-SC-FDMA system in Fig.1, only the DFT and IDFT blocks at the transmitter and receiver are replaced by the DCT and IDCT blocks, respectively.

The DCT of a sampled signal $x_{n}$ can be written as follows [28]:

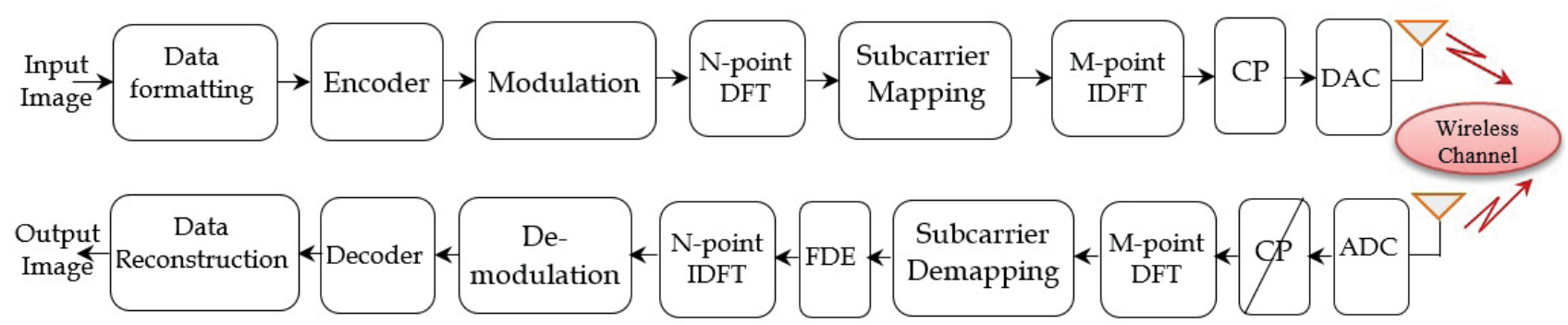

Fig. 1 The schematic diagram of the Conventional SC-FDMA system

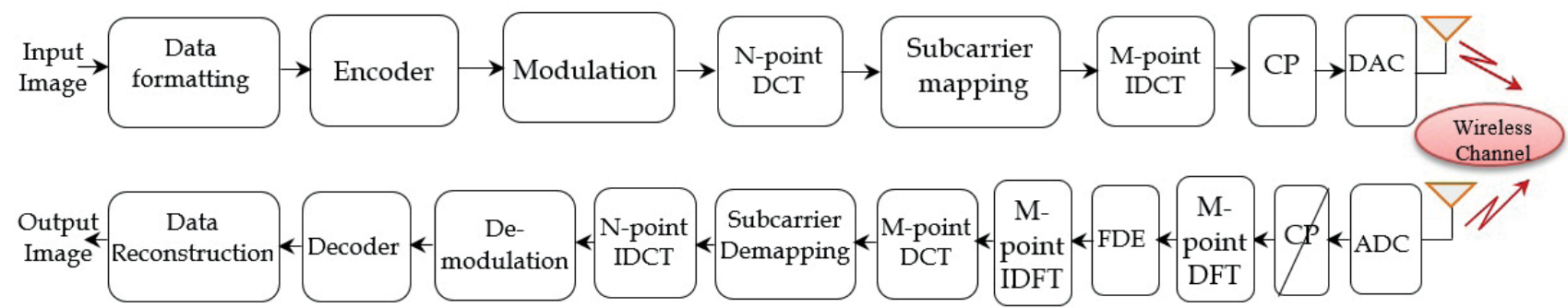

Fig. 2 The schematic diagram of DCT SC-FDMA system 
$X_{k}=\sqrt{\frac{2}{N}} \beta_{k} \sum_{n=0}^{N-1} x_{n} \cos \left(\frac{\pi k(2 n+1)}{2 N}\right), k=0, \ldots, N-1$

where $X_{k}$ is the modulated complex symbol, $N$ is the input block size and $\beta_{k}$ can be expressed as:

$\beta_{k}=\left\{\frac{1}{\sqrt{2}}\right.$ for $k=0 \quad 1$ for $k=1, \ldots, N-1$.

The discrete time signal can be reconstruction via the inverse discrete cosine transform (IDCT) using Eq. (5) [28]:

$y_{m}=\sqrt{\frac{2}{M}} \sum_{l=0}^{M-1} Y_{l} \beta_{l} \cos \left(\frac{\pi l(2 m+1)}{2 N}\right), l=0, \ldots, N-1$,

where $Y_{l}$ represents the signal after the mapping process, $M=Q . N$ represents the number of subcarriers and $Q$ represents the maximum number of simultaneous users that the system supports.

\section{2D Chaotic baker map algorithm}

Encryption can be considered as a method of protecting information from unauthorized access by converting it into a non-recognizable form to prevent illegal access to this information. It mainly involves scrambling of data contents such as text, images, video, etc., resulting in new format of data which is unreadable during transmission. Several chaos-based image encryption methods have been proposed in recent years [25-27]. Chaotic maps is one of the most important methods used for securing digital images, which are transmitted over the different wireless networks. The main advantage of these methods is the efficiency in image encryption due to excellent properties such as ergodicity and high sensitivity to their initial conditions and control parameters. The 2D Chaotic Baker map in its discretized version is a good candidate for image encryption. The signal samples can be arranged into a $2 \mathrm{D}$ format and then randomized using the chaotic Baker map. The Chaotic Baker map generates permuted sequences with lower correlation between their samples and adds a level of encryption to the transmitted signal. The discretized Baker map is an efficient tool for randomizing items in a square matrix. To encrypt an image of size $(M \times M)$, the 2D Chaotic Baker map in its discretized version can be used for this purpose. Let $B\left(m_{1}, \ldots, m_{k}\right)$, represents the discretized Baker map, where the vector of secret key $S_{k e y}=\left[m_{1}, \ldots, m_{k}\right]$, contains of $k$ integer elements, and it is chosen such that each integer $m_{i}$ divides $(M)$, and $\left(m_{1}+\ldots+m_{k}=M\right)$ where $M$ is defined as the number of data items in one row

Let $M_{i}=m_{1}+\ldots+m_{k}$, the pixel at the indices $(r, s)$ is mapped to the indices [29].

$M_{\left(m_{1}, \ldots, m_{k}\right)}(r, s)=\left[\begin{array}{l}\frac{M}{m_{i}}\left(r-M_{i}\right)+s \bmod \frac{M}{m_{i}}, \\ \frac{m}{M}\left(s-s \bmod \frac{M}{m_{i}}\right)+M_{i}\end{array}\right]$,

with $M_{i} \leq r \leq M_{i}+m_{i}$ and $0 \leq s \leq M$.

The discretized versions of baker map of an $(M \times M)$ square matrix is performed using the following steps:

1. Division of an $(M \times M)$ square matrix into $k$ rectangles of width $m_{i}$ and with height $M$.

2. Each rectangle $M \times m_{i}$ is rearranged to a row in the novel permuted rectangle.

3. The scan of rectangles starts from left to right beginning with upper rectangles then lower ones.

4. In each rectangle, the scan of its elements starts from the bottom left corner towards upper elements.

For more explanation, an example illustrative of the permutation induced by the discretized versions of the Baker map for an $(8 \times 8)$ square matrix is shown in Fig. 3, with $(M=8)$ and the secret key $S_{k e y}=\left[m_{1}, m_{2}, m_{3}\right]=[2,4,2]$.

\begin{tabular}{|l|l|llll|lll|}
\hline $\mathrm{P}_{1}$ & $\mathrm{P}_{2}$ & $\mathrm{P}_{3}$ & $\mathrm{P}_{4}$ & $\mathrm{P}_{5}$ & $\mathrm{P}_{6}$ & $\mathrm{P}_{7}$ & $\mathrm{P}_{8}$ \\
\hline $\mathrm{P}_{9}$ & $\mathrm{P}_{10}$ & $\mathrm{P}_{11}$ & $\mathrm{P}_{12}$ & $\mathrm{P}_{13}$ & $\mathrm{P}_{14}$ & $\mathrm{P}_{15}$ & $\mathrm{P}_{16}$ \\
\hline $\mathrm{P}_{17}$ & $\mathrm{P}_{18}$ & $\mathrm{P}_{19}$ & $\mathrm{P}_{20}$ & $\mathrm{P}_{21}$ & $\mathrm{P}_{22}$ & $\mathrm{P}_{23}$ & $\mathrm{P}_{24}$ \\
\hline $\mathrm{P}_{25}$ & $\mathrm{P}_{26}$ & $\mathrm{P}_{27}$ & $\mathrm{P}_{28}$ & $\mathrm{P}_{29}$ & $\mathrm{P}_{30}$ & $\mathrm{P}_{31}$ & $\mathrm{P}_{32}$ \\
\hline $\mathrm{P}_{33}$ & $\mathrm{P}_{34}$ & $\mathrm{P}_{35}$ & $\mathrm{P}_{36}$ & $\mathrm{P}_{37}$ & $\mathrm{P}_{38}$ & $\mathrm{P}_{39}$ & $\mathrm{P}_{40}$ \\
\hline $\mathrm{P}_{41}$ & $\mathrm{P}_{42}$ & $\mathrm{P}_{43}$ & $\mathrm{P}_{44}$ & $\mathrm{P}_{45}$ & $\mathrm{P}_{46}$ & $\mathrm{P}_{47}$ & $\mathrm{P}_{48}$ \\
\hline $\mathrm{P}_{49}$ & $\mathrm{P}_{50}$ & $\mathrm{P}_{51}$ & $\mathrm{P}_{52}$ & $\mathrm{P}_{53}$ & $\mathrm{P}_{54}$ & $\mathrm{P}_{55}$ & $\mathrm{P}_{56}$ \\
\hline $\mathrm{P}_{57}$ & $\mathrm{P}_{58}$ & $\mathrm{P}_{59}$ & $\mathrm{P}_{60}$ & $\mathrm{P}_{61}$ & $\mathrm{P}_{62}$ & $\mathrm{P}_{63}$ & $\mathrm{P}_{64}$ \\
\hline
\end{tabular}

(a)

\begin{tabular}{|l|l|l|l|l|l|l|l|}
\hline $\mathrm{P}_{31}$ & $\mathrm{P}_{23}$ & $\mathrm{P}_{15}$ & $\mathrm{P}_{7}$ & $\mathrm{P}_{32}$ & $\mathrm{P}_{24}$ & $\mathrm{P}_{16}$ & $\mathrm{P}_{8}$ \\
\hline $\mathrm{P}_{63}$ & $\mathrm{P}_{55}$ & $\mathrm{P}_{47}$ & $\mathrm{P}_{39}$ & $\mathrm{P}_{64}$ & $\mathrm{P}_{56}$ & $\mathrm{P}_{48}$ & $\mathrm{P}_{40}$ \\
\hline $\mathrm{P}_{11}$ & $\mathrm{P}_{3}$ & $\mathrm{P}_{12}$ & $\mathrm{P}_{4}$ & $\mathrm{P}_{13}$ & $\mathrm{P}_{5}$ & $\mathrm{P}_{14}$ & $\mathrm{P}_{6}$ \\
\hline $\mathrm{P}_{27}$ & $\mathrm{P}_{19}$ & $\mathrm{P}_{28}$ & $\mathrm{P}_{20}$ & $\mathrm{P}_{29}$ & $\mathrm{P}_{21}$ & $\mathrm{P}_{30}$ & $\mathrm{P}_{22}$ \\
\hline $\mathrm{P}_{43}$ & $\mathrm{P}_{35}$ & $\mathrm{P}_{44}$ & $\mathrm{P}_{36}$ & $\mathrm{P}_{45}$ & $\mathrm{P}_{37}$ & $\mathrm{P}_{46}$ & $\mathrm{P}_{38}$ \\
\hline $\mathrm{P}_{59}$ & $\mathrm{P}_{51}$ & $\mathrm{P}_{60}$ & $\mathrm{P}_{52}$ & $\mathrm{P}_{61}$ & $\mathrm{P}_{53}$ & $\mathrm{P}_{62}$ & $\mathrm{P}_{54}$ \\
\hline $\mathrm{P}_{25}$ & $\mathrm{P}_{17}$ & $\mathrm{P}_{9}$ & $\mathrm{P}_{1}$ & $\mathrm{P}_{26}$ & $\mathrm{P}_{18}$ & $\mathrm{P}_{10}$ & $\mathrm{P}_{2}$ \\
\hline $\mathrm{P}_{57}$ & $\mathrm{P}_{49}$ & $\mathrm{P}_{41}$ & $\mathrm{P}_{33}$ & $\mathrm{P}_{58}$ & $\mathrm{P}_{50}$ & $\mathrm{P}_{42}$ & $\mathrm{P}_{34}$ \\
\hline
\end{tabular}

(b)

Fig. 3 Mechanism of 2D Chaotic baker map operation for an $8 \times 8$ square matrix with the secret $S_{k e y}=[2,4,2]$; (a) The $8 \times 8$ original matrix divided into 8 rectangles, (b) encrypted version of matrix after applying discretized Baker map 
The secret key $S_{k e y}$ is generated according to the processed image size. For example, we assume the dimensions of the image are $(M \times M)$ and the secret key is $\left(m_{1}, m_{2}, m_{3}, \ldots, m_{k}\right)$. It is a set of $k$ numbers, its sum equal to $(M)$, such as $\left(m_{1}+m_{2}+m_{3}+\ldots+m_{k}=M\right)$.

\section{Proposed DWT-Based SC-FDMA system for Encrypted Image Transmission}

DWT has found high-popularity for the application of signal processing and communications due to its advantages of effectiveness, robustness, and low-complexity. Compared to the traditional DFT and DCT, the DWT has the ability to multiresolution analysis of signals with better localization in time and frequency, and then is able to reduce multipath effects and interference. DWT can be performed to decompose the signal into approximation coefficients and detail coefficients [30]. In general, the implementation of DWT is achieved by the means of analysis and synthesis filters, such as the low-pass filters, generate approximation coefficients, while the high-pass filters generate detail coefficients.

The block diagram of the proposed wavelet transform-based SC-FDMA (DWT-SC-FDMA) scheme for encrypted image transmission is shown in Fig. 4. In this section, a brief overview of the different blocks of the proposed transmission system is presented. Therefore, the architecture of the DWT-based SC-FDMA scheme for image transmission is similar to the DCT-based SC-FDMA. As shown in Fig. 2, only the IDCT and the DCT blocks are replaced by inverse DWT (IDWT) and DWT blocks, respectively.

At the transmitter side, the grayscale image to be transmitted is transformed into a binary 2D form and then encrypted using the 2D Chaotic Baker map algorithm. The discretized version of the 2D Chaotic Baker map is applied to randomize the items in a square matrix. After the randomization process, the binary data will be encoded using a convolutional encoder with rate of $1 / 2$, and then modulated using quadrature-phase shift keying (QPSK) modulation. Let $x_{n}$ denote the modulated data symbols. Then, we can describe the signal after the DWT as follows [31]:

$$
X_{k}^{m}=\sum_{n=0}^{N-1} x_{n} 2^{\frac{k}{2}} \psi\left(2^{k}-m\right),
$$

where $X_{k}^{m}$ denote the wavelet coefficients and $\psi(t)$ denote the wavelet basis function.

After the DWT block, the mapping process is performed, which assigns frequency domain symbols to subcarriers. After that, the IDWT is applied to transform the frequency symbols into the time domain prior to the transmission. After the IDWT processing, a CP is added to prevent inter-block interference (IBI) due to multi-path environments. Finally, the digital signal is converted into analog form by the DAC and then transmitted through the wireless channel.

At the receiver side, the $\mathrm{CP}$ is removed from the received signal and the signal is then transformed into the frequency domain using DFT, followed by the FDE processes. The IDFT at the receiver transforms the equalized samples into the time domain. After the IDFT, the samples are transformed into the frequency domain via the DWT. After the subcarriers de-mapping process, which isolates the frequency domain samples of each signal, an IDWT is applied. After IDWT processing, the demodulation and the decoding processes are applied to restore data bits. The obtained binary bits are reshaped to a square matrix and then the 2D Chaotic Baker map decryption is applied to this matrix. Finally, the image data reconstruction stage at the receiver, which used for converting the binary format to restore the original image.

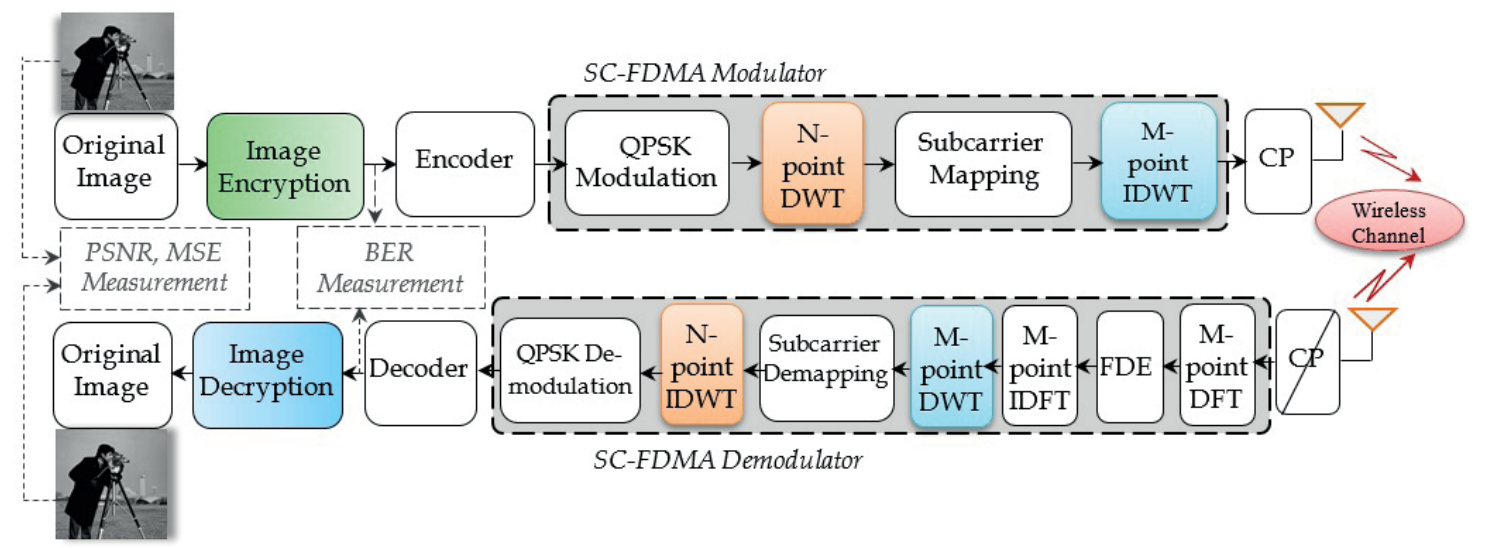

Fig. 4 The schematic diagram of the proposed DWT-based SC-FDMA scheme for encrypted image transmission 
The Haar wavelet is used in our simulations because it is the simplest type of wavelet and serves as a prototype for all other wavelet transforms. To provide a good tradeoff between the performance of encrypted image transmission and implementation complexity at a low level, we will only use the two-level Haar wavelet transform in the decomposition and reconstruction stages.

\section{Simulation results}

\subsection{Simulation parameters}

Extensive simulations have been carried out to investigate and evaluate the performance of encrypted image transmission over three different versions of SC-FDMA-based systems. The 2D Chaotic baker map algorithm is applied in this system to encrypt the image before transmission, using the simulation parameters given in Table 1. Six grayscale images (Cameraman, Lena, Barbara, Bird, Peppers, and House $)$ of size $(256 \times 256)$ are considered as input to the simulation framework. The original image is first encrypted by 2D Chaotic baker map and then transmitted at different SNR scenarios through the conventional DFTSC-FDMA, DCT-SC-FDMA, and DWT-SC-FDMA systems. To test the efficiency of the proposed scheme, different channel models are considered in this simulation, such as the AWGN channel, Vehicular A, and SUI-3 channels. The performance of the proposed scheme is evaluated by the means of the BER and the PSNR metrics. The most used metric to measure the fidelity of the reconstructed images at the receiver is the PSNR, which can be written as:

$P S N R=10 \log _{10} \frac{[255]^{2}}{M S E}$,

where MSE denotes the mean squared error between the reconstructed and the original images, which is given by:

$$
M S E=\frac{1}{M \times M} \sum_{i=0}^{M} \sum_{j=0}^{M}[f(i, j)-\hat{f}(i, j)]^{2},
$$

where, $f(i, j)$ and $\hat{f}(i, j)$ are the original and reconstructed images of sizes $(M \times M)$, respectively.

\subsection{PSNR performance}

In this section, the grayscale image is used as input to the simulation framework, such as the Cameraman image of size $256 \times 256$ is encrypted by a $2 \mathrm{D}$ Chaotic baker map and transmitted through the conventional SC-FDMA, DCTSC-FDMA, and DWT-SC-FDMA systems with QPSK modulation over different wireless channels. The transmitted and encrypted images are shown in Figs. 5 and 6,
Table 1 Simulation parameter of SC-FDMA system parameters

\begin{tabular}{lc}
\hline Simulation Parameter & Value \\
\hline FFT size & 512 symbols \\
Input block size & 128 symbols \\
Cyclic prefix size & 20 samples \\
Images size & $256 \times 256$ \\
Channel coding & Convolutional code with rate $1 / 2$ \\
Modulation type & QPSK \\
Subcarriers mapping & Interleaved and localized \\
Channel model & AWGN, SUI-3, Vehicular A \\
Noise environment & AWGN \\
Equalizer type & MMSE equalizer \\
\hline
\end{tabular}

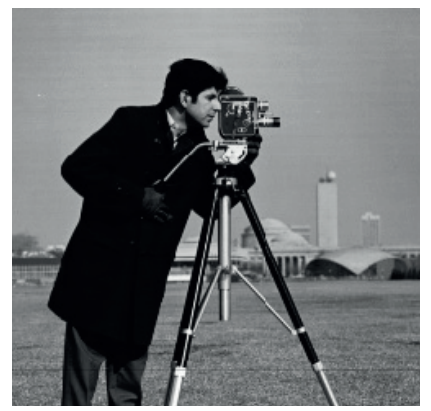

Fig. 5 Original image

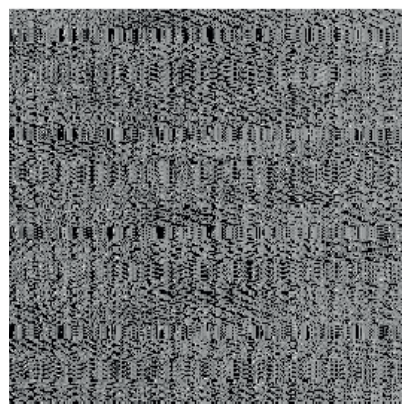

Fig. 6 Encrypted image with 2D Chaotic backer map respectively. The PSNR values of the received image are calculated for different SNR values from 0 through $10 \mathrm{~dB}$ in $2 \mathrm{~dB}$ steps. The wireless channels considered in this paper are the AWGN channel, Vehicular A, and SUI-3 channels.

Figs. 7(a)-(f) illustrate a visual comparison between reconstructed Cameraman images transmitted over the different SC-FDMA versions in the presence of the AWGN channel at $\mathrm{SNR}=5 \mathrm{~dB}$. The original Cameraman image is first encrypted using the 2D Chaotic Baker map algorithm and then transmitted through one of the SC-FDMA-based systems such as DFT-SC-FDMA or DCT-SC-FDMA or DWT-SC-FDMA systems. The results of this scenario for encrypted images transmission demonstrate that the DWT-SC-FDMA approach has higher PSNR values when compared to the other SC-FDMA-based systems. It is also clear that the DWT-SC-FDMA scheme enhances the PSNR performance of reconstructed images after decryption.

In addition, the DWT-IFDMA scheme gives a better PSNR performance than DWT-LFDMA as can be seen from Figs. 7(a)-(f), because the IFDMA scheme is more robust to multi-path fading.

Figs. 8(a)-(f) illustrate a visual comparison between reconstructed Cameraman images transmitted over the different SC-FDMA versions in the case of the Vehicular A 


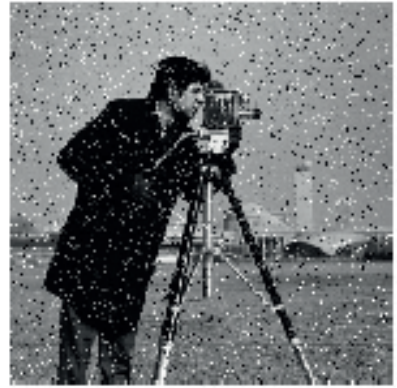

(a)

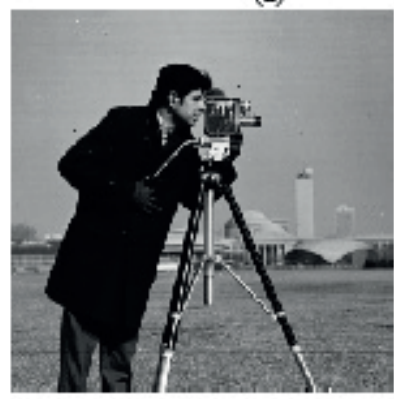

(c)

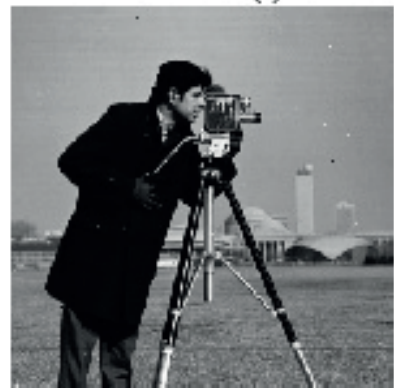

(e)

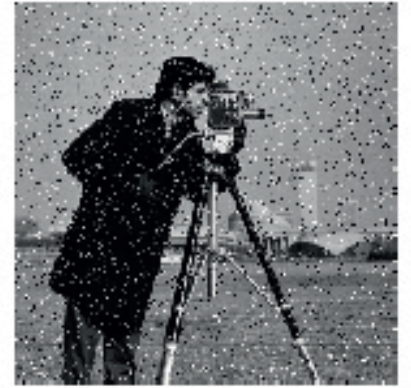

(b)

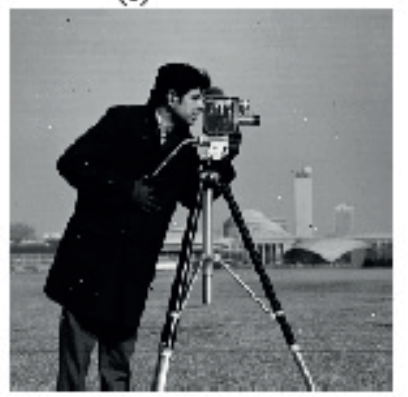

(d)

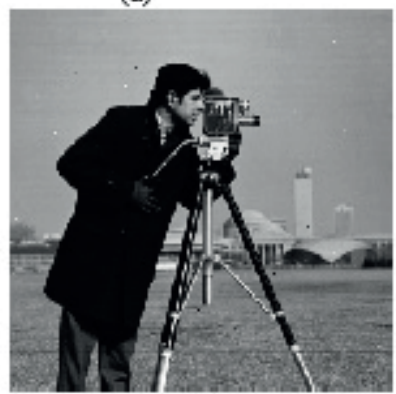

(f)
Fig. 7 Reconstructed 2D Chaotic-encrypted Cameraman images after decryption for AWGN channel at $\mathrm{SNR}=5 \mathrm{~dB}$ in the cases: (a) DFTIFDMA, PSNR $=19.00 \mathrm{~dB}$, (b) DFT-LFDMA,PSNR $=18.93 \mathrm{~dB}$,

(c) Z DCT-IFDMA, PSNR $=41.40 \mathrm{~dB}$, (d) DCT-LFDMA, $P S N R=39.87 \mathrm{~dB}$. (e) DWT-IFDMA, PSNR $=43.19 \mathrm{~dB}$, (f) DWTLFDMA, PSNR $=42.39 \mathrm{~dB}$, chains, respectively

channel at $\mathrm{SNR}=5 \mathrm{~dB}$. As we can observe, there is significant distortion for the received image due to the Doppler frequency and multi-path delay in a channel.

The experimental results confirm the effectiveness of the proposed approach in the encrypted image transmission quality over LTE standard based-on SC-FDMA.

Fig. 9 shows a comparison between the PSNR performances of the 2D Chaotic-encrypted Cameraman image transmitted over DWT SC-FDMA systems for two subcarriers mapping schemes and different wireless channels at various SNR scenarios. As shown in the figure, the PSNR performance of the proposed wavelet transform-based scheme for the two subcarriers mapping modes (IFDMA and LFDMA) in presence of an AWGN channel is almost the same. The simulation results when applying the Vehicular A and SUI-3 channels, the PSNR performance of DWT-IFDMA is always better than the DWT-LFDMA.

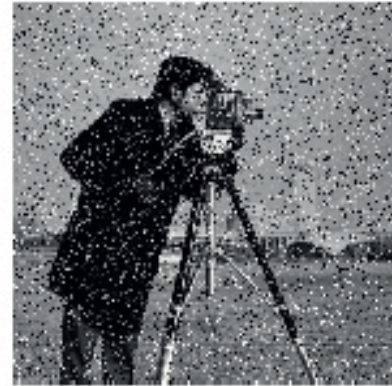

(a)

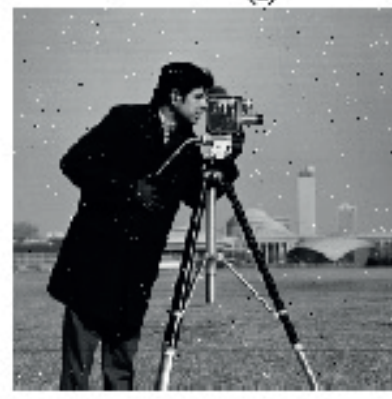

(c)

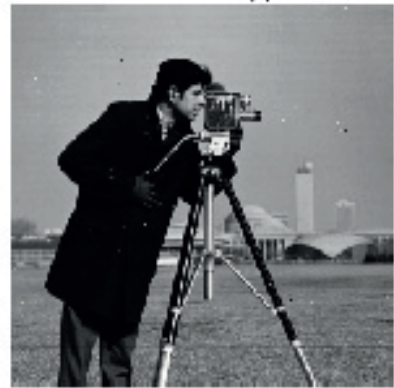

(e) (b)

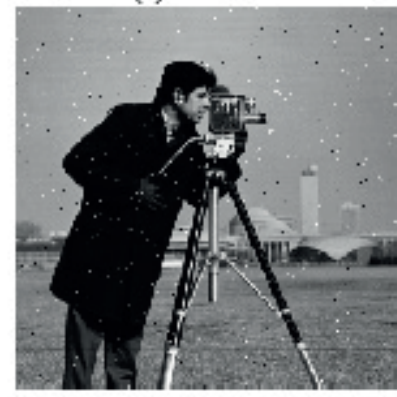

(d)

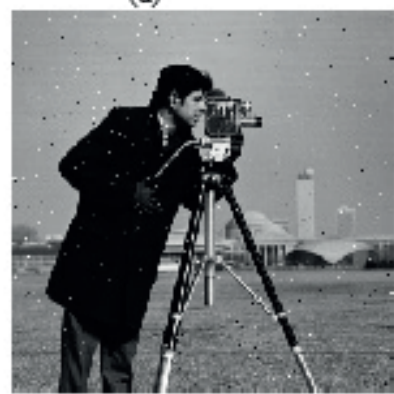

(f)

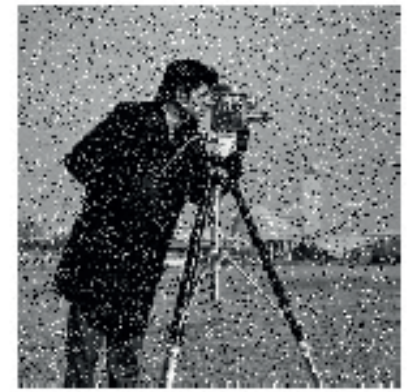

Fig. 8 Reconstructed 2D Chaotic-encrypted Cameraman images after decryption for Vehicular A channel at $\mathrm{SNR}=5 \mathrm{~dB}$ in the cases: (a) DFT-

IFDMA, PSNR $=16.34 \mathrm{~dB}$, (b) DFT-LFDMA, PSNR $=16.37 \mathrm{~dB}$,

(c) DCT-IFDMA, PSNR $=30.08 \mathrm{~dB}$, (d) DCT-LDMA, PSNR $=29.12 \mathrm{~dB}$, (e) DWT-IFDMA, PSNR $=42.43 \mathrm{~dB}$, (f) DWT-LFDMA, PSNR $=30.48 \mathrm{~dB}$, chains, respectively

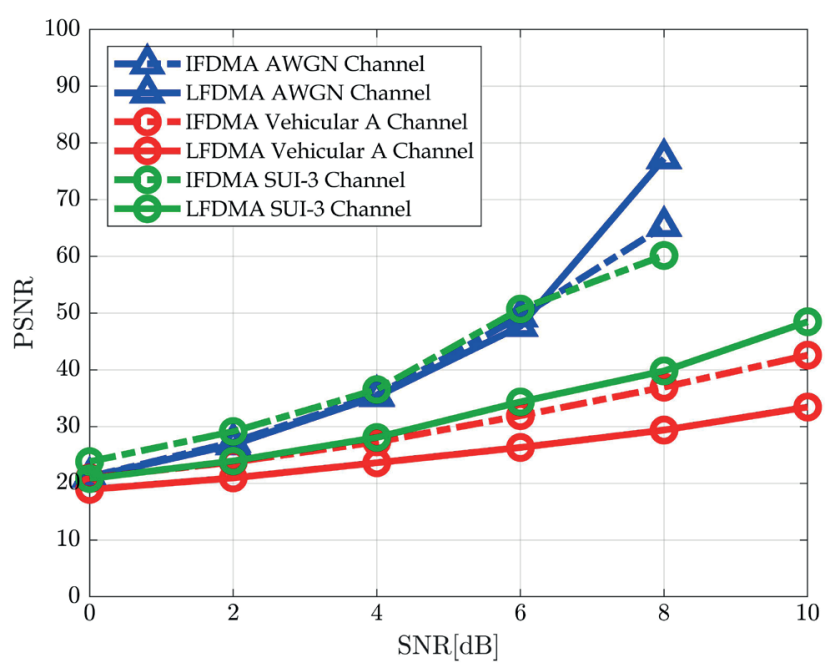

Fig. 9 PSNR performance of the 2D Chaotic-encrypted Cameraman image transmitted with the proposed DWT-SC-FDMA scheme over different wireless channels 
Fig. 10 illustrates the PSNR performance of the transmission of the 2D Chaotic-encrypted Cameraman image through the above-mentioned two SC-FDMA-based systems and the proposed DWT-SC-FDMA system over an AWGN channel. From this figure, it is observed that the PSNR values for the three systems are increased by increasing the SNR values. Furthermore, it is clear that the DWT-SC-FDMA system significantly outperforms both the DCT-SC-FDMA and DCT-SC-FDMA systems in terms of PSNR at all SNR values. For clarity, at SNR $=8 \mathrm{~dB}$, the proposed scheme can achieve a significant PSNR performance improvement of about 32 and $26 \mathrm{~dB}$ for the DFTLFDMA and DCT-LFDMA schemes, respectively. Hence, the wavelet-based SC-FDMA system can provide a considerable improvement in PSNR performance when compared to conventional systems.

The same previous scenario is given when the Vehicular A channel is used and the results are presented in Fig. 11. It is clear that the DWT SC-FDMA system enhances the PSNR performance of the encrypted image transmission by about 18 and $27 \mathrm{~dB}$ at SNR=10dB for the DCT-IFDMA and the DFT-IFDMA schemes, respectively. These results demonstrate that the wavelet transform-based SC-FDMA system for encrypted image transmission achieves PSNR values higher than the conventional DFT-SC-FDMA and DCT-SC-FDMA systems.

Tables 2 and 3 summarize a comparison between the obtained PSNR values of the received images at different SNR values, when the encrypted Cameraman image is transmitted with the above-mentioned three SC-FDMA-based systems over AWGN and SUI-3 channels, respectively.

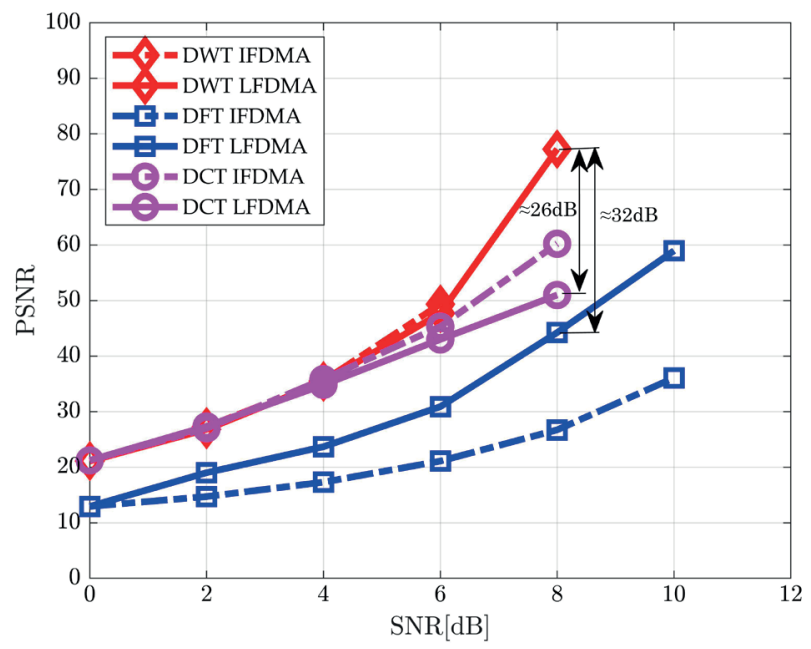

Fig. 10 PSNR performance of the 2D Chaotic-encrypted Cameraman image transmitted with the three different versions of SC-FDMA systems over AWGN channel

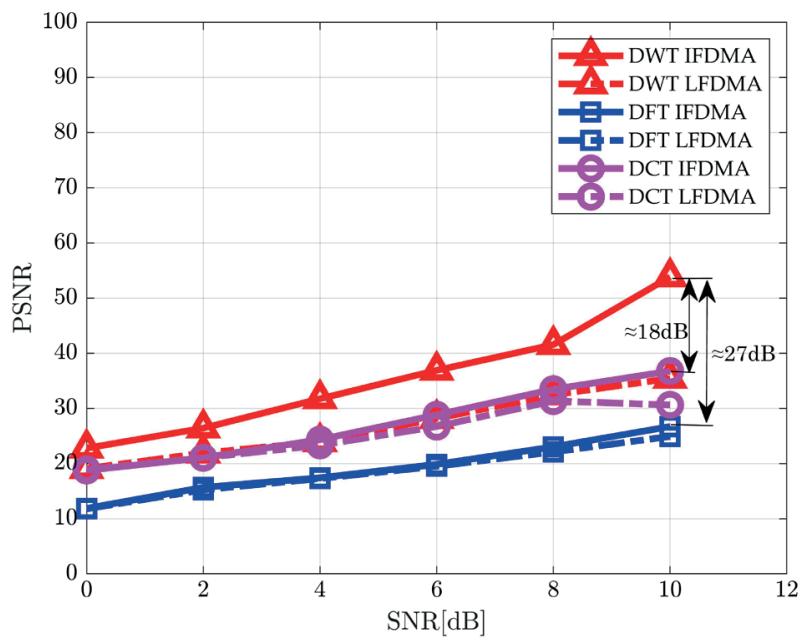

Fig. 11 PSNR performance of the 2D Chaotic-encrypted Cameraman image transmitted with the three different versions of SC-FDMA over Vehicular A channel

Table 2 PSNR values of reconstructed Cameraman image over AWGN channel

\begin{tabular}{lcccccc}
\hline $\begin{array}{l}\text { SNR } \\
{[\mathrm{dB}]}\end{array}$ & \multicolumn{2}{c}{ DFT SC-FDMA } & \multicolumn{2}{c}{ DCT SC-FDMA } & \multicolumn{2}{c}{ DWT SC-FDMA } \\
\hline 0 & 12.93 & 12.93 & 21.22 & 21.22 & 21.13 & 21.08 \\
2 & 14.71 & 19.01 & 26.98 & 27.35 & 27.25 & 26.81 \\
4 & 17.30 & 23.68 & 35.84 & 34.82 & 35.54 & 35.32 \\
6 & 21.09 & 30.89 & 45.33 & 43.00 & 49.36 & 47.70 \\
8 & 26.72 & 44.23 & 60.23 & 51.00 & Inf & 77.26 \\
10 & 36.08 & 58.97 & Inf & Inf & Inf & 77.26 \\
\hline
\end{tabular}

Table 3 PSNR values of reconstructed Cameraman image over SUI-3 channel

\begin{tabular}{ccccccc}
\hline $\begin{array}{c}\text { SNR } \\
\text { [dB] }\end{array}$ & \multicolumn{2}{c}{ DFT-SC-FDMA } & \multicolumn{2}{c}{ DCT SC-FDMA } & \multicolumn{2}{c}{ DWT SC-FDMA } \\
0 & 12.32 & 12.37 & 17.87 & 16.83 & 20.18 & 17.68 \\
2 & 13.52 & 13.54 & 21.08 & 19.28 & 24.6 & 20.86 \\
4 & 15.28 & 15.22 & 24.88 & 22.72 & 31.04 & 24.49 \\
6 & 17.61 & 17.49 & 29.88 & 25.85 & 38.99 & 29.30 \\
8 & 20.41 & 19.94 & 36.41 & 30.32 & 48.18 & 34.99 \\
10 & 24.46 & 23.96 & 40.13 & 32.79 & 60.0 & 38.42 \\
\hline
\end{tabular}

Table 4 summarizes the obtained PSNR values of the received images with the three different versions of the SC-FDMA system considered in this paper for the transmission of the 2D Chaotic-encrypted images over the SUI-3 channel at $\mathrm{SNR}=8 \mathrm{~dB}$.

\subsection{BER performance}

Fig. 12 illustrates the BER performance of the wavelet transform-based SC-FDMA system over different wireless 
Table 4 PSNR values of reconstructed images with the different version of SC-FDMA systems for the transmission of the 2D Chaotic-encrypted images over SUI-3 channel at SNR $=8 \mathrm{~dB}$

\begin{tabular}{lcccccc}
\hline \multirow{2}{*}{ Image } & \multicolumn{2}{c}{ DFT SC-FDMA } & \multicolumn{2}{c}{ DCT SC-FDMA } & \multicolumn{2}{c}{ DWT SC-FDMA } \\
& IFDMA & LFDMA & IFDMA & LFDMA & IFDMA & LFDMA \\
\hline $\begin{array}{l}\text { Camera- } \\
\text { man }\end{array}$ & 20.54 & 20.53 & 40.65 & 36.79 & 81.24 & 39.39 \\
Lena & 20.61 & 20.29 & 41.32 & 36.97 & 54.15 & 40.76 \\
Barbara & 20.56 & 20.42 & 43.21 & 37.92 & 54.08 & 40.72 \\
Bird & 20.70 & 20.53 & 40.58 & 36.36 & 54.08 & 42.48 \\
Peppers & 20.65 & 20.46 & 42.54 & 38.17 & 60.17 & 41.47 \\
House & 20.71 & 20.34 & 42.08 & 36.11 & 60.17 & 40.46 \\
\hline
\end{tabular}

channels for the transmission of encrypted Cameraman image. The gray-scale Cameraman image is first encrypted by a $2 \mathrm{D}$ Chaotic baker map algorithm and then transmitted over different wireless channels at different SNR values. From this figure, it is clear that the performance of the DWT-SC-FDMA system in the case of the AWGN channel is the best as compared to the other wireless channels used in our simulations, such as Vehicular A, and SUI-3 channels. This is due to wireless channel degradations such as multi-path fading and interference that affect the performance of SC-FDMA-based systems when the encrypted image is transmitted.

Fig. 13 depicts a comparison between the BER performance of the wavelet transform-based SC-FDMA, DFT-SCFDMA and DCT-SC-FDMA systems for the transmission of 2D Chaotic-encrypted Cameraman image over AWGN channel. It is clear that the wavelet-based system outperforms traditional SC-FDMA systems. It can achieve an improvement of about $0.5 \mathrm{~dB}$ and $3.5 \mathrm{~dB}$ in SNR at BER $=10^{-4}$ for the DCT-IFDMA and DFT-IFDMA scheme, respectively.

Fig. 14 depicts a comparison between the BER performance of the DWT-SC-FDMA, DFT-SC-FDMA, and DCTSC-FDMA systems for the transmission of 2D Chaoticencrypted Cameraman image over Vehicular A wireless channel. We observe in Fig. 14 that the wavelet transform-based system provides better performance than the conventional SC-FDMA systems. It can give an improvement of about $2 \mathrm{~dB}$ and $4 \mathrm{~dB}$ in SNR at BER $=10^{-4}$ for the DCT-IFDMA and the DFT-IFDMA schemes, respectively.

The same previous scenario is given when the SUI-3 wireless channel is used and the results are presented in Fig. 15. It is clear that the wavelet-based SC-FDMA system provides good BER performance when compared to the DCT-SC-FDMA and DFT-SC-FDMA systems, in particular at high SNR values.

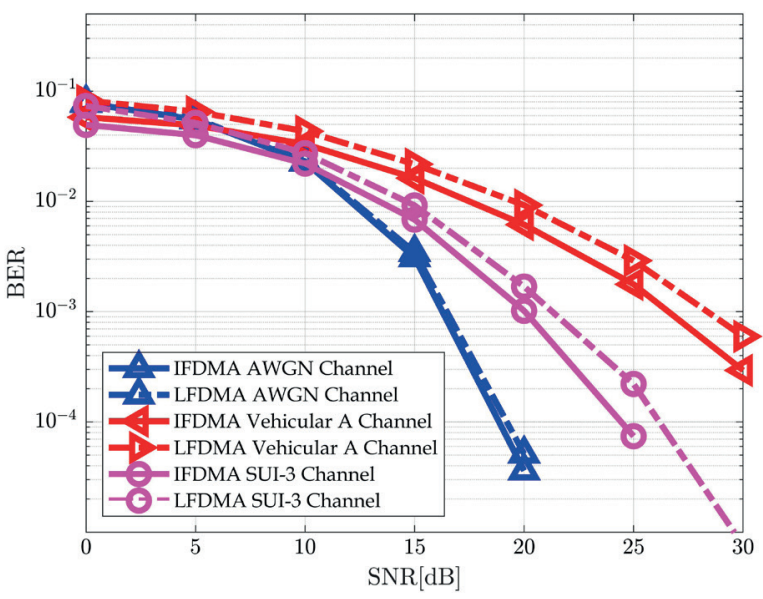

Fig. 12 BER performance of the DWT SC-FDMA system for the transmission of 2D Chaotic-encrypted Cameraman image over different wireless channels

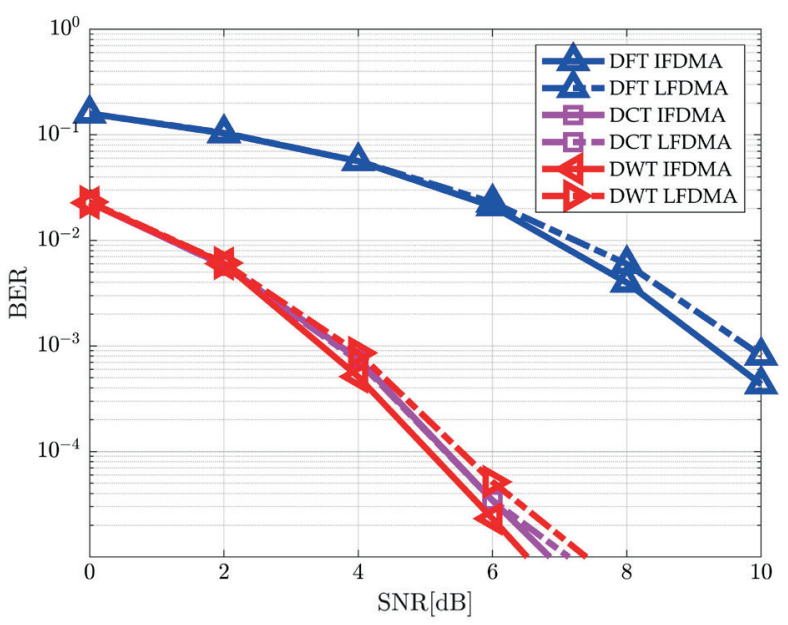

Fig. 13 Comparison of BER performance among DFT-SC-FDMA, DCT SC-FDMA and DWT SC-FDMA systems for the transmission of 2D Chaotic-encrypted Cameraman image over AWGN channel

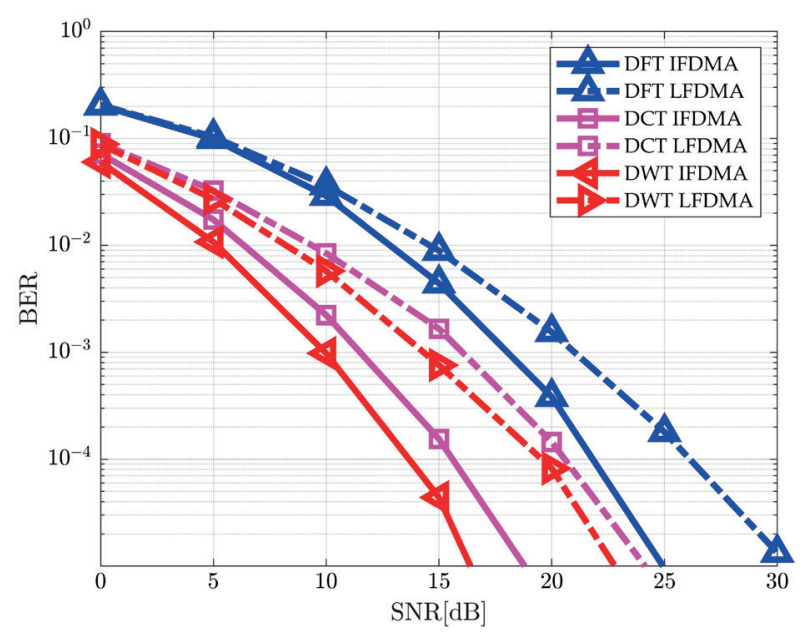

Fig. 14 Comparison of BER performance among DFT SC-FDMA, DCT SC-FDMA and DWT SC-FDMA systems for the transmission of 2D Chaotic-encrypted Cameraman image over Vehicular A channel 


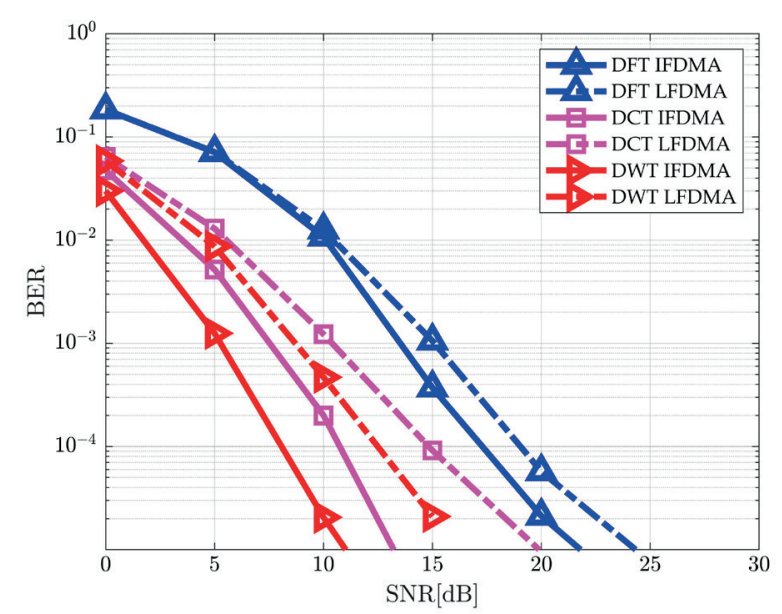

Fig. 15 Comparison of BER performance among DFT-SC-FDMA, DCTSC-FDMA and DWT-SC-FDMA systems for the transmission of 2D Chaotic-encrypted Cameraman image over SUI-3 channel

From the obtained results of these simulations, we notice that the BER performance of the proposed DWT SC-FDMA system is the best as compared to the DCTSC-FDMA and DFT-SC-FDMA systems for all wireless channels used in our simulation. Therefore, the proposed

\section{References}

[1] Myung, H. G., Goodman, D. J. "Single Carrier FDMA: A New Air Interface for Long Term Evaluation", John Wiley \& Sons Ltd., New York, NY, USA, 2008.

https://doi.org/10.1002/9780470758717

[2] Myung, H. G., Lim, J., Goodman, D. J. "Single carrier FDMA for uplink wireless transmission", IEEE Vehicular Technology Magazine, 1(3), pp. 30-38, 2006.

https://doi.org/10.1109/mvt.2006.307304

[3] Al-Kamali, F. S., Dessouky, M. I., Sallam, B. M., Shawki, F., Abd El-Samie, F. E. "A new single carrier FDMA system based on the discrete cosine transform", In: International Conference on Computer Engineering \& Systems, (ICCES), Cairo, Egypt, 2009, pp. $555-560$.

https://doi.org/10.1109/ICCES.2009.5383071

[4] Elbehery, A., Abdelwahab, S. A. S., El Naby, M. A., Hassan, E. S., Elaraby, S. "Image Transmission with DCT Based SC-FDMA System Using Continuous Phase Modulation", In: 30th National Radio Science Conference (NRSC), Cairo, Egypt, 2013, pp. 149-157. https://doi.org/10.1109/NRSC.2013.6587937

[5] Abd El-Samie, F. E., Al-Kamali, F. S., Al-Nahari, A. Y., Dessouky, M. I. "SC-FDMA for Mobile Communications", CRC Press, Boca, Raton, FL, USA, 2013. https://doi.org/10.1201/b15157

[6] Shukur, M. A. N., Pahwa, K., Singhal, A. "Implementing SC-FDMA and OFDMA in MATLAB", International Journal of Computing and Corporate Research, 3(6), pp. 1-21, 2013. [online] Available at: http://www.ijccr.com/November2013/5.pdf [06 November 2021] scheme is the most suitable for the effective transmission of encrypted images with SC-FDMA and is more robust to interference and the effects of multi-path fading.

\section{Conclusion}

This paper presents an efficient scheme for the transmission of encrypted images over SC-FDMA systems based on the discrete cosine transform (DWT). The proposed approach exploits wavelet transform analysis and the 2D Chaotic Baker map to improve the performance of SC-FDM for uplink communications. The performance of 2D Chaotic-encrypted image transmission over the DWT SC-FDMA system has been investigated for different wireless channels. In addition, a comparison study between the DWT-SC-FDMA system, DFT-SC-FDMA system and DCT-SC-FDMA system has been presented to demonstrate the effectiveness of the wavelet transform in encrypted image transmission. Simulation results verify that the DWT SC-FDMA system can achieve a significant improvement in both PSNR and BER performance, with sufficient robustness to the effects of the wireless channel.

[7] 3rd Generation Partnership Project (3GPP) "LTE; Evolved Universal Terrestrial Radio Access (E-UTRA). Physical channels and modulation (3GPP TS 36.211 version 11.5.0 Release 11)", [pdf] ETSI, Sophia Antipolis, Cedex, France. Available at: https:/www.etsi.org/deliver/ etsi_ts/136200_136299/136211/11.05.00_60/ts_136211v110500p. pdf [12 January 2021]

[8] Al-Kamal, F. S., Hassan, E. S., Abd El-Naby, M., Shawki, F., El-Khamy, S. E., Dessouky, M. I., Sallam, B. M., Alshebeili, S. A., Abd El-samie, F. E. "An Efficient Transceiver Scheme for SC-FDMA Systems Based on Discrete Wavelet Transform and Discrete Cosine Transform", Wireless Personal Communications, 83(4), pp. 3133-3155, 2015.

https://doi.org/10.1007/s11277-015-2587-8

[9] Mehallel, E., Abed, D., Boukaache, A., Bouchemel, A. "Enhancement of image transmission using chaotic interleaving with discrete wavelet transform-based single-carrier frequency division multiple access system", International Journal of Communication Systems, 34(7), e4728, 2021.

https://doi.org/10.1002/dac.4728

[10] Abdel Hamid, Z., Samir, M., Abd El-atty, S. M., El-Hennawy, A. E., El Shenawy, H., Alshebeili, S. A., Abd El-Samie, F. E. "On the Performance of FFT/DWT/DCT-based OFDM Systems with Chaotic Interleaving and Channel Estimation Algorithms", Wireless Personal Communications, 78(2), pp.1495-1510, 2014. https://doi.org/10.1007/s11277-014-1830-z

[11] Bouchemel, A., Abed, D., Moussaoui, A. "Enhancement of Compressed Image Transmission in WMSNs Using Modified $\mu$-Nonlinear Transformation", IEEE Communications Letters, 22(5), pp. 934-937, 2018.

https://doi.org/10.1109/LCOMM.2018.2812821 
[12] Eldokany, I., El-Rabaie, E. M., Elhalafawy, S. M., Zein Eldin, M. A., Shahieen, M. H., Soliman, N. F., ..., Abd El-Samie, F. E. "Efficient Transmission of Encrypted Images with OFDM in the Presence of Carrier Frequency Offset", Wireless Personal Communications, 84(1), pp. 475-521, 2015. https://doi.org/10.1007/s11277-015-2645-2

[13] Soliman, N. F., Hassan, E. S., Shaalan, A. H. A., Fouad, M. M., El-Khamy, S. E., Albagory, Y., ..., Abd El Samie, F. E. "Efficient Image Communication in PAPR Distortion Cases", Wireless Personal Communications, 83(4), pp. 2773-2834, 2015. https://doi.org/10.1007/s11277-015-2568-y

[14] El-Bakary, E. M., Hassan, E. S., Zahran, O., El-Dolil, S. A., Abd El-Samie, F. E. "Efficient Image Transmission with Multi-Carrier CDMA", Wireless Personal Communications, 69(2), pp. 979-994, 2013. https://doi.org/10.1007/s11277-012-0622-6

[15] Al-Kamali, F. S., Qasem, A., Abuasbaa, S. A., Qasem, G. A. "SC-FDMA and OFDMA: An Efficient Wireless Image Transmission Schemes", Journal of Control and Systems Engineering, 4(1), pp. 74-83, 2016. https://doi.org/10.18005/JCSE0401008

[16] Al-kamali F. S., Al-Fuhaidy F. A., Al-soufy K. A. "Wireless Image Transmission over Frequency Selective Channel Using Recent OFDMA Systems", American Journal of Computation, Communication and Control,5(11), pp. 30-38, 2018. [online] Available at: http://article.aascit.org/file/pdf/9010956.pdf [22 June 2020]

[17] Elbehery, A., Abdelwahab, S. A. S., El Naby, M. A., Hassan, E. S., Elaraby S. "C31. Efficient image transmission over the single carrier frequency division multiple access system using chaotic interleaving", In: 29th National Radio Science Conference (NRSC), Cairo, Egypt, 2012, pp. 411-420. https://doi.org/10.1109/NRSC.2012.6208549

[18] Padmapriya, V. M., Thenmozhi, K., Praveenkumar, P., Amirtharajan, R. "ECC joins first time with SC-FDMA for Mission "security"", Multimedia Tools and Application, 79(25-26), pp. 17945-17967, 2020. https://doi.org/10.1007/s11042-020-08610-5

[19] Han, F., Yu, X., Han, S. "Improved Baker map for image encryption", In: 1st International Symposium on Systems and Control in Aerospace and Astronautics, (ISSCAA), Harbin, China, pp. 12731276, 2006.

https://doi.org/ 10.1109/ISSCAA.2006.1627519
[20] Zhang, L., Liao, X., Wang, X. "An image encryption approach based on chaotic maps", Chaos, Solitons \& Fractals, 24(3), pp. 759-765, 2005.

https://doi.org/10.1016/j.chaos.2004.09.035

[21] Chen, G., Mao, Y., Chui, C. K. "A symmetric encryption scheme based on 3D chaotic cat maps", Chaos, Solitons \& Fractals, 21(3), pp. 749-761, 2004 https://doi.org/10.1016/j.chaos.2003.12.022

[22] Kocarev, L., Jakimoski, G., Stojanovski, T., Parlitz, U. "From chaotic maps to encryption schemes", In: IEEE International Symposium on Circuits and Systems, Monterey, CA, USA, 1998, pp. 514-517. https://doi.org/10.1109/ISCAS.1998.698968

[23] Baptista, M. S. "Cryptography with chaos", Physics Letters A, 240(1-2), pp. 50-54, 1998. https://doi.org/10.1016/S0375-9601(98)00086-3

[24] Kocarev, L., Jakimoski, G. "Logistic map as a block encryption algorithm", Physics Letters A, 289(4-5), pp. 199-206, 2001. https://doi.org/10.1016/S0375-9601(01)00609-0

[25] Pareek, N. K., Patidar, V., Sud, K. K. "Cryptography using multiple one-dimensional chaotic maps", Communications Nonlinear Science and Numerical Simulation, 10(7), pp. 715-723, 2005. https://doi.org/10.1016/j.cnsns.2004.03.006

[26] Xiang, T., Wong, K-W., Liao, X. "A novel symmetrical cryptosystem based on discretized two-dimensional chaotic map", Physics Letters A, 364(3-4), pp. 252-258, 2007. https://doi.org/10.1016/j.physleta.2006.12.020

[27] Mao, Y., Chen, G., Lian, S. "A novel fast image encryption scheme based on 3D chaotic baker maps", International Journal of Bifurcation and Chaos, 14(10), pp. 3613-3624, 2004. https://doi.org/10.1142/S021812740401151X

[28] Tan, P., Beaulieu, N. C. "A Comparison of DCT-Based OFDM and DFT-Based OFDM in Frequency Offset and Fading Channels", IEEE Transactions on Communications, 54(11), pp. 2113-2125, 2006. https://doi.org/10.1109/TCOMM.2006.884852

[29] Oppenheim, A. V., Schafer, R. W., Buck, J. R. "Discrete-Time Signal Processing", Prentice Hall, New York, NY, USA, 1998.

[30] Fridrich, J. "Symmetric Ciphers Based on Two-Dimensional Chaotic Maps", International Journal of Bifurcation and Chaos in Applied Sciences and Engineering, 8(6), pp. 1259-1284, 1998. https://doi.org/10.1142/S021812749800098X

[31] Skodras, A. N. "Discrete Wavelet Transform: An Introduction", Hellenic Open University, Patras, Greece, Rep. HOU-CS-TR-200302-EN, 2003. 